\title{
Precision Measurement of the First Ionization Potential of Nobelium
}

\author{
P. Chhetri, ${ }^{1,2, *}$ D. Ackermann, ${ }^{2,3}$ H. Backe, ${ }^{4}$ M. Block ${ }^{2,5,6}$ B. Cheal, ${ }^{7}$ C. Droese,${ }^{8}$ Ch. E. Düllmann, ${ }^{2,5,6}$ J. Even, ${ }^{6,9}$ \\ R. Ferrer, ${ }^{10}$ F. Giacoppo, ${ }^{2,6}$ S. Götz, ${ }^{2,5,6}$ F. P. Heßberger, ${ }^{2,6}$ M. Huyse, ${ }^{10}$ O. Kaleja, ${ }^{2,11, \dagger}$ J. Khuyagbaatar, ${ }^{2,6}$ P. Kunz, ${ }^{12}$ \\ M. Laatiaoui, ${ }^{2,6}$ F. Lautenschläger, ${ }^{1,2}$ W. Lauth, ${ }^{4}$ N. Lecesne, ${ }^{3}$ L. Lens, ${ }^{2,5}$ E. Minaya Ramirez, ${ }^{13}$ A. K. Mistry, ${ }^{2,6}$ \\ S. Raeder, ${ }^{2,6}$ P. Van Duppen, ${ }^{10}$ Th. Walther, ${ }^{1}$ A. Yakushev, ${ }^{2,6}$ and Z. Zhang ${ }^{14}$ \\ ${ }^{1}$ Institut für Angewandte Physik, Technische Universität Darmstadt, Schlossgartenstrasse 7, D-64289 Darmstadt, Germany \\ ${ }^{2}$ GSI Helmholtzzentrum für Schwerionenforschung GmbH, Planckstrasse 1, D-64291 Darmstadt, Germany \\ ${ }^{3}$ Grand Accélérateur National d'Ions Lourds, Bd Henri Becquerel, BP 55027-14076 Caen Cedex 05, France \\ ${ }^{4}$ Institut für Kernphysik, Johannes Gutenberg-Universität Mainz, Johann-Joachim-Becher Weg 45, D 55128 Mainz, Germany \\ ${ }^{5}$ Institut für Kernchemie, Johannes Gutenberg-Universität, Fritz-Strassmann Weg 2, D-55128 Mainz, Germany \\ ${ }^{6}$ Helmholtz-Institut Mainz, Staudingerweg 18, D-55128 Mainz, Germany \\ ${ }^{7}$ Department of Physics, Oxford Street, University of Liverpool, L69 7ZE Liverpool, United Kingdom \\ ${ }^{8}$ Institut für Physik, Universität Greifswald, Felix-Hausdorff-Strasse 6, D-17489 Greifswald, Germany \\ ${ }^{9}$ KVI-Center for Advanced Radiation Technology, Rijksuniversiteit Groningen, Zernikelaan 25, 9747 AA Groningen, Netherlands \\ ${ }^{10}$ KU Leuven, Instituut voor Kern- en Stralingsfysica, Celestijnenlaan 200D, B-3001 Leuven, Belgium \\ ${ }^{11}$ Institut für Kernphysik, Technische Universität Darmstadt, Schlossgartenstrasse 9, D-64289 Darmstadt, Germany \\ ${ }^{12}$ TRIUMF, 4004 Wesbrook Mall, Vancouver, British Columbia V6T 2A3, Canada \\ ${ }^{13}$ Institut de Physique Nucléaire Orsay, 15 rue Georges Clemenceau, 91406 Orsay, France \\ ${ }^{14}$ Institute of Modern Physics, Chinese Academy of Sciences, 509 Nanchang Road, 730000 Lanzhou, China
}

(Received 13 April 2018; published 29 June 2018)

\begin{abstract}
One of the most important atomic properties governing an element's chemical behavior is the energy required to remove its least-bound electron, referred to as the first ionization potential. For the heaviest elements, this fundamental quantity is strongly influenced by relativistic effects which lead to unique chemical properties. Laser spectroscopy on an atom-at-a-time scale was developed and applied to probe the optical spectrum of neutral nobelium near the ionization threshold. The first ionization potential of nobelium is determined here with a very high precision from the convergence of measured Rydberg series to be $6.62621 \pm 0.00005 \mathrm{eV}$. This work provides a stringent benchmark for state-of-the-art many-body atomic modeling that considers relativistic and quantum electrodynamic effects and paves the way for highprecision measurements of atomic properties of elements only available from heavy-ion accelerator facilities.
\end{abstract}

DOI: 10.1103/PhysRevLett.120.263003

To date, 118 chemical elements are known [1]. Their chemical behavior is mainly determined by the electronic configuration. This is subject not only to the Coulomb interaction, but, with increasing atomic number $Z$, also to large electron-electron correlations, quantum electrodynamic (QED) effects, and relativistic effects. These relativistic effects influence the binding energy of the valence electrons, thus the chemical properties, as they cause a shrinking of $s$ and $p_{1 / 2}$ electron orbitals and change the screening of the Coulomb potential by the inner-shell electrons [2]. Currently, many-body methods such as multiconfiguration Dirac-Fock (MCDF), relativistic coupled cluster (RCC), and configuration interaction (CI) are employed to study these effects [3-6]. This has motivated many gas- and liquid-phase chemistry experiments, which could be performed in the region of the heaviest elements [7-9]. The first ionization potential (IP) represents the binding energy of the most weakly bound electron of an atom. An accurate determination of the IP provides a crucial test for our understanding of the electronic structure and the chemical properties of an element. According to the actinide concept put forward by Seaborg [10], the actinide series consists of 15 elements starting from actinium (Ac, $Z=89$ ) and ending with lawrencium ( $\mathrm{Lr}, Z=103)$. To date, the IP of 12 elements in the actinide series has been determined using resonance ionization mass spectroscopy, Rydberg convergence, and surface ionization techniques [11-17]. $\mathrm{Lr}$ is the heaviest element for which the IP has been experimentally determined using a surface ionization technique [17] with a precision of about $0.08 \mathrm{eV}$ and potentially susceptible to unknown systematic effects. Laser spectroscopy offers a far greater precision, in the $\mu \mathrm{eV}$ regime, but its application to the heaviest elements is hampered by the absence of known atomic transitions and the need for element synthesis from nuclear fusion reactions utilizing large accelerator facilities. 
Recently, we reported the development of a technique sensitive enough for laser spectroscopic studies of nobelium $(Z=102)$ on an atom-at-a-time scale [18]. While this marked a first foray of optical spectroscopy into the region of the transfermium $(Z>100)$ elements by addressing the difficulties mentioned above, an ambiguity due to an interatomic quenching process in the nobelium atom led to significant uncertainties for the element's IP $[18,19]$. Here we present the results of extended measurements that overcome these limitations of our previous work and which have greatly opened up the versatility of the technique. Two complementary methods have since been developed, whereby excitation to Rydberg states (prior to ionization) proceeds (i) via the direct excitation from an intermediate atomic state, or (ii) following buffer gas collisional deexcitation to an intermediate excited state. The first has now enabled the measurement of the IP of nobelium with a precision 3 orders of magnitude greater than that previously reported [18]. For the second, we here exploit a complementary process to determine the electronic energy levels also when the ground state optical transition is forbidden.

The experiment was performed behind the velocity filter SHIP at GSI, Darmstadt, Germany [20]. The isotope ${ }^{254}$ No (half-life $T_{1 / 2}=51 \mathrm{~s}$ ) was produced in the completefusion evaporation reaction ${ }^{208} \mathrm{~Pb}\left({ }^{48} \mathrm{Ca}, 2 \mathrm{n}\right){ }^{254} \mathrm{No}$ with a cross section of $2 \mu \mathrm{b}$ [21]. The lead sulfide target had an average thickness of $470 \mu \mathrm{g} / \mathrm{cm}^{2}$. The ${ }^{48} \mathrm{Ca}$ beam from the UNILAC accelerator exhibited a macropulse structure of $5 \mathrm{~ms}$ beam-on and $15 \mathrm{~ms}$ beam-off period. The beam intensity at a projectile energy of $4.55 \mathrm{AMeV}$ [22] was typically 0.7 particle $\mu \mathrm{A}$ (about $4 \times 10^{12}$ particles per second). The beam was additionally gated in accordance with user-defined measurement cycles. The rate of fusion products delivered to the experiment was checked by a retractable position-sensitive 16-strip silicon detector placed at the focal plane of SHIP. The fusion products were separated from the primary beam by the velocity filter SHIP and thermalized inside an optical gas cell, filled with 95 mbar of ultrahigh purity argon. A $3.5 \mu \mathrm{m}$ thick aluminized Mylar entrance foil separated the optical cell from the high vacuum of SHIP. The thermalized fusion products that remained charged were collected on a tantalum filament of $125 \mu \mathrm{m}$ diameter. After a collection time of $25 \mathrm{~s}$ [23], optimized for the half-life of ${ }^{254} \mathrm{No}$, the incoming beam was switched off for $5 \mathrm{~s}$ during which the filament was pulse heated for $300 \mathrm{~ms}$ to about $1350 \mathrm{~K}$ in order to evaporate the collected fusion products as neutral atoms. The temperature of the filament was monitored by a fast infrared pyrometer (LumaSense, IMPAC IS 6 Advanced). A two-step photoionization process was then employed to ionize the atoms.
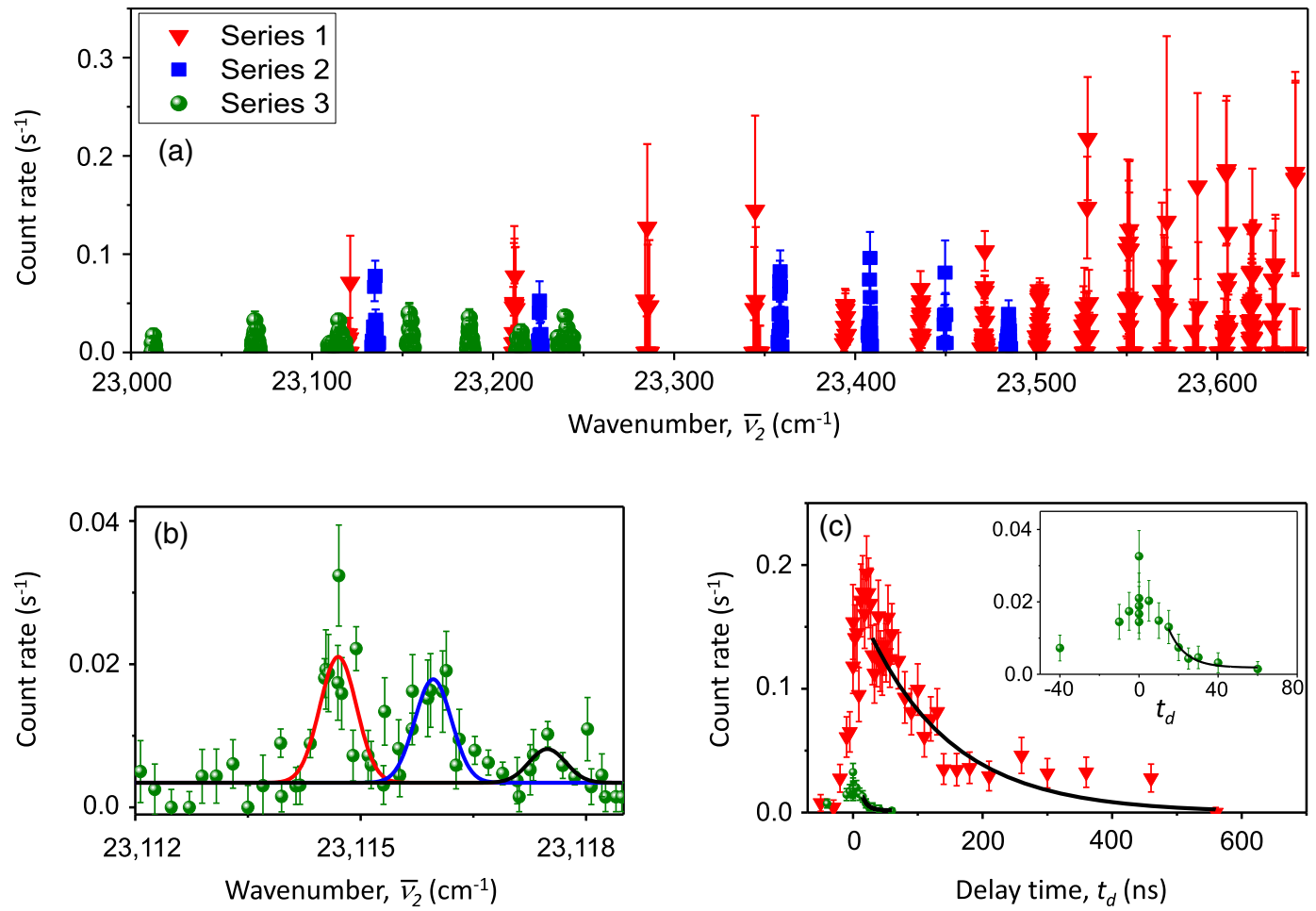

FIG. 1. (a) Scan of the second-step excitation $\bar{\nu}_{2}$ in ${ }^{254}$ No. The first step was fixed at $\bar{\nu}_{1}=29961.457 \mathrm{~cm}^{-1}$. Series 1 and 2 were measured at a argon gas pressure of 95 mbar. Series 3 was measured at a pressure of 65 mbar, to reduce the buffer gas collision induced quenching. (b) A selected Rydberg resonance proceeding from the intermediate state with Gaussian fits (solid lines) to the data. (c) Resonance ionization signal for delayed Rydberg excitation, series 1 (red points) and 3 (green points), proceeding via the intermediate states. The solid line indicates an exponential fit to the data with lifetime $\tau$ about $100 \mathrm{~ns}$ (series 1 and 2 ) and $5 \mathrm{~ns}$ (series 3 ). Inset: magnification of delayed ionization of series 3. The $\alpha$-decay count rates are normalized to a primary beam current of 1 particle $\mu \mathrm{A}$. 
The produced ions were transported by electric fields onto a silicon detector where they were identified by their characteristic $\alpha$-decay energy. The laser light was provided by two tunable dye lasers (Lambda Physik FL and LPD series) pumped by two excimer lasers with a $100 \mathrm{~Hz}$ repetition rate at 248 and $308 \mathrm{~nm}$, respectively. The dye laser pulses had a pulse duration of $18 \mathrm{~ns}$ with a jitter of about $10 \mathrm{~ns}$. The wavelengths were continuously monitored with a wavelength meter (HighFinesse-Angstrom, WS/7-UVU) that was calibrated to an internal neon lamp. A more detailed description of the experimental setup and method can be found in Refs. [24-26].

A two-color photon-excitation scheme was used to search for Rydberg states in ${ }^{254} \mathrm{No}$. We operated one dye laser at $\bar{\nu}_{1}=29961.457 \mathrm{~cm}^{-1}$ initiating in a first step the ${ }^{1} S_{0} \rightarrow{ }^{1} P_{1}$ ground state transition [18], while another synchronized dye laser provided tunable light for the second excitation step, $\bar{\nu}_{2}$. The atoms that were successfully excited into a Rydberg state were subsequently ionized either by residual laser light, black-body radiation, or collisional processes. In total, a spectral range of the second excitation step from 23000 to $23650 \mathrm{~cm}^{-1}$ was scanned to locate 29 Rydberg states. At a buffer gas pressure of 95 mbar two different series comprising 22 Rydberg states were identified as shown in Fig. 1(a) (series 1 and 2), significantly adding to those reported previously [18]. However, in this previous work it could not be unambiguously determined from which state the second excitation step originated, due to the possibility that gas induced quenching (following excitation to the ${ }^{1} P_{1}$ intermediate state) populates close-lying long-lived states. Reducing the buffer gas pressure to about 65 mbar, helped to observe another series [series 3 in Fig. 1(a)] with a substantially reduced count rate. The lowest-lying Rydberg states from this series showed resolved multiplet structures [Fig. 1(b)], which can be attributed to different fine structure components. The splitting of the multiplets decreases with increasing principal quantum number.

In order to identify the state from which the second transition takes place, the $\alpha$ signal at resonance was measured as a function of time delay between the two laser pulses. The first step was kept at $\bar{\nu}_{1}=29961.457 \mathrm{~cm}^{-1}$ populating the ${ }^{1} P_{1}$ state and the second step was set to excite a specific Rydberg state. This measurement allows us to probe the lifetime of the intermediate state which enables the disentanglement of the different Rydberg series [see Fig. 1(c)]. Members of one series (3) indicate a short-lived intermediate state with a lifetime of about $5 \mathrm{~ns}$ in agreement with the expectations for the ${ }^{1} P_{1}$ state. The intermediate state for the members of the other two series ( 1 and 2$)$ has a longer lifetime of about $100 \mathrm{~ns}$ proving the involvement of an additional intermediate level populated by buffer gas induced quenching of the ${ }^{1} P_{1}$ state.

In the data analysis of the individual resonances, we used a $\chi^{2}$ minimization routine to extract the relevant parameters.
We determined the excitation energy of the Rydberg states from the centroids of single or multiple Gaussian fits to each resolved state or unresolved multiplet, respectively. The excitation energy of the Rydberg states follow a trend given by the Rydberg formula [27]

$$
h \bar{\nu}_{2}(n)=h \bar{\nu}_{\text {lim }}-R_{m} /[n-\delta(n)]^{2},
$$

where $h \bar{\nu}_{\text {lim }}=$ IP $-E_{\text {interm }}$ denotes the ionization limit for an intermediate state of energy $\mathrm{E}_{\text {interm }} h$ is the Planck constant, $n$ is the principal quantum number of the valence electron, and $R_{m}$ is the reduced mass Rydberg constant for ${ }^{254}$ No. The quantum defect $\delta(n)$ can be parametrized by the Ritz expansion [28]

$$
\delta(n)=\delta_{0}+\sum_{i=1} B_{i} /\left(n-\delta_{0}\right)^{2 \cdot i}
$$

where $\delta_{0}$ and $B_{i}$ are the fitting parameters. Figure 2 (upper panel) shows the position of all the observed peaks as a function of their principal quantum number $(n)$. The assignment of $n$ was obtained by restricting the quantum defect $\delta(n)$ between 0 and 1. However, this choice of $\delta(n)$ does not affect the resulting convergence limits. The best fit according to Eq. (1) to the series associated with the Rydberg states proceeding from the ${ }^{1} P_{1}$ state converged to a value $\bar{\nu}_{\text {lim }}=23482.14 \pm 0.27 \mathrm{~cm}^{-1}$. The presence of the buffer gas induces a shift in the energy of the Rydberg

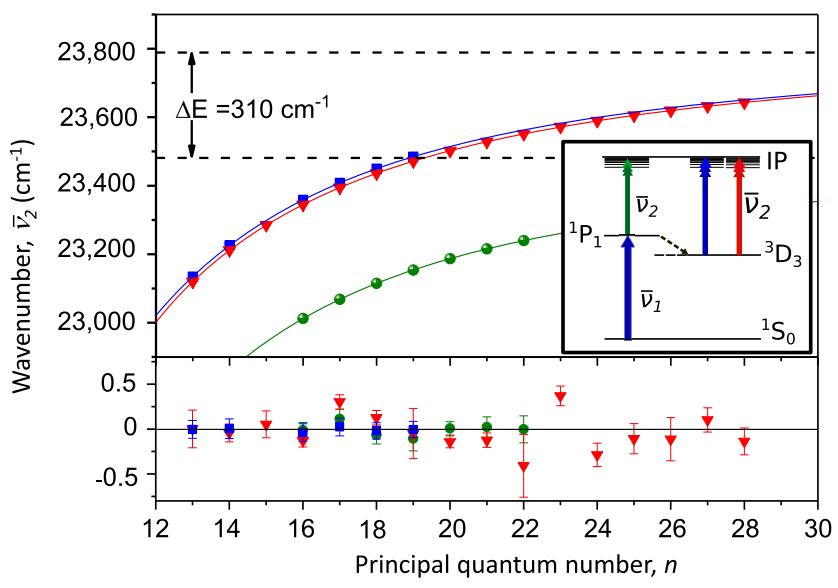

FIG. 2. Analysis of the Rydberg states. Top panel: the position of all observed Rydberg states as a function of the principal quantum number $n$. The Rydberg-Ritz formula, Eq. (1), was fitted to the center position of the peaks and the main peaks of the multiplets belonging to different $n$. The dashed lines indicate the convergence limits of the individual series. Here, the gap between them corresponds to the energy difference between the intermediate $\left({ }^{1} P_{1}\right.$ and $\left.{ }^{3} D_{3}\right)$ states. Inset: effective level scheme for Rydberg excitation of ${ }^{254}$ No. Lower panel: residuals of the fits. The uncertainty of the residuals includes the statistical uncertainty $(1 \sigma)$ from the fit as well as the systematic uncertainties $(1 \sigma)$ from the wavelength determination. 
TABLE I. Experimental values of the first IP of nobelium and energy of the ${ }^{3} \mathrm{D}_{3}$ level, compared with: IHFSCC, MCDF, $\mathrm{CI}+$ all orders. The uncertainties are based on predictions + estimates like made in Ref. [18].

\begin{tabular}{lll}
\hline \hline Method & \multicolumn{1}{c}{$\mathrm{IP}\left(\mathrm{cm}^{-1}\right)$} & ${ }^{3} \mathrm{D}_{3}\left(\mathrm{~cm}^{-1}\right)$ \\
\hline Experiment (this work) & $53444.0 \pm 0.4$ & $29652_{-1}^{+8}$ \\
IHFSCC [4] & $53489 \pm 800$ & $29897 \pm 800$ \\
CI+ all orders [5] & $54390 \pm 1100$ & $30183 \pm 1100$ \\
MCDF [6] & $53701 \pm 1100$ & \\
Extrapolation [30] & $53600 \pm 600$ & \\
\hline \hline
\end{tabular}

states and thus in the extracted IP. Hence, after the correction arising from the pressure, which is about $-0.0060(7) \mathrm{cm}^{-1} / \mathrm{mbar}$ for the homologue element ytterbium $(\mathrm{Yb}, Z=70$ ) [29], we obtain a first ionization potential for nobelium to IP $=53444.0 \pm 0.4 \mathrm{~cm}^{-1}$ corresponding to $6.62621 \pm 0.00005 \mathrm{eV}$. Predictions of the IP of nobelium using different theoretical models [4-6,30] are given in Table I for comparison along with the experimental value obtained in this work.

The other two series (1 and 2), converged to a mean value of $\bar{\nu}_{\text {lim }}=23792_{-8}^{+1} \mathrm{~cm}^{-1}$ when taking higher-order corrections to the Ritz expansion [Eq. (2)] into account. Comparing the convergence limits of the series, it becomes clear that series 1 and 2 proceed from a state located about $310 \mathrm{~cm}^{-1}$ below the ${ }^{1} P_{1}$ state. A likely assignment for this level at an energy of $29652_{-1}^{+8} \mathrm{~cm}^{-1}$ is the ${ }^{3} D_{3}$ state, predicted to be 159 [4] or $20 \mathrm{~cm}^{-1}$ [5] below the ${ }^{1} P_{1}$ state. Other lower-lying $D$ states are predicted to be more than $1000 \mathrm{~cm}^{-1}$ below the ${ }^{1} P_{1}$ state. The results have been summarized in Table I.

An assignment for each series can be obtained from the quantum defect and by comparing our results for nobelium with literature values for $\mathrm{Yb}$. Figure 3 shows the measured quantum defect $\delta(n)$ in $\mathrm{Yb}$ and No as a function of the principal quantum number. The quantum defect values for $\mathrm{Yb}$ were calculated using the energy levels from references [31,32]. Series 1 and 2 in No show a similar $l$ dependent quantum defect constant $\delta_{0}$ of about 0.9 and 0.1 as the $n p$ and $n f$ series in $\mathrm{Yb}$ (Fig. 3, left panel), respectively, and matches with the expectation [33]. The quantum defect of series 1 features a unique trend probably due to the presence of high-lying perturbing orbitals interfering with the Rydberg series, similar to those observed for the $n p$ series in $\mathrm{Yb}$ [32]. In the evaluation for extracting the IP this can be treated perturbatively by using higher order corrections in the Ritz expansion given in Eq. (2). Series 3, with $\delta_{0} \approx 0.7$ is similar to the $n d$ series in $\mathrm{Yb}$. Based on these observations an assignment of $n p$, $n f$, and $n d$ is proposed for series 1, 2, and 3, respectively. According to these observations, series 1 and 2 are likely to proceed via a $D$ state, substantiating the assignment of the intermediate state.

In conclusion, the observed high-lying Rydberg states in nobelium enabled the determination of the first IP and the level energy of the ${ }^{3} D_{3}$ state with high precision. In general, we found a good agreement between the predictions and our results. In particular, the intermediate Hamiltonian Fock-space coupled-cluster (IHFSCC) and configuration interaction method combined with the linearized singledouble coupled-cluster $(\mathrm{CI}+$ all order) calculations deviate by less than $2 \%$ from the experimental value for the IP. In the case of the ${ }^{3} D_{3}$ state, the deviation is larger compared to other levels potentially due to correlation effects, underestimation of QED effects, or both. Our values serve as a benchmark for the treatment of relativistic, QED, and electron correlation effects in state-of-the-art calculations predicting atomic properties of the heaviest elements. Our measurements show that nobelium exhibits the highest measured first IP in the actinide series [11,17]. In analogy to ytterbium, its lanthanide homologue, this corresponds to closed $5 f$ and $7 s$ atomic shells in nobelium. Experimental

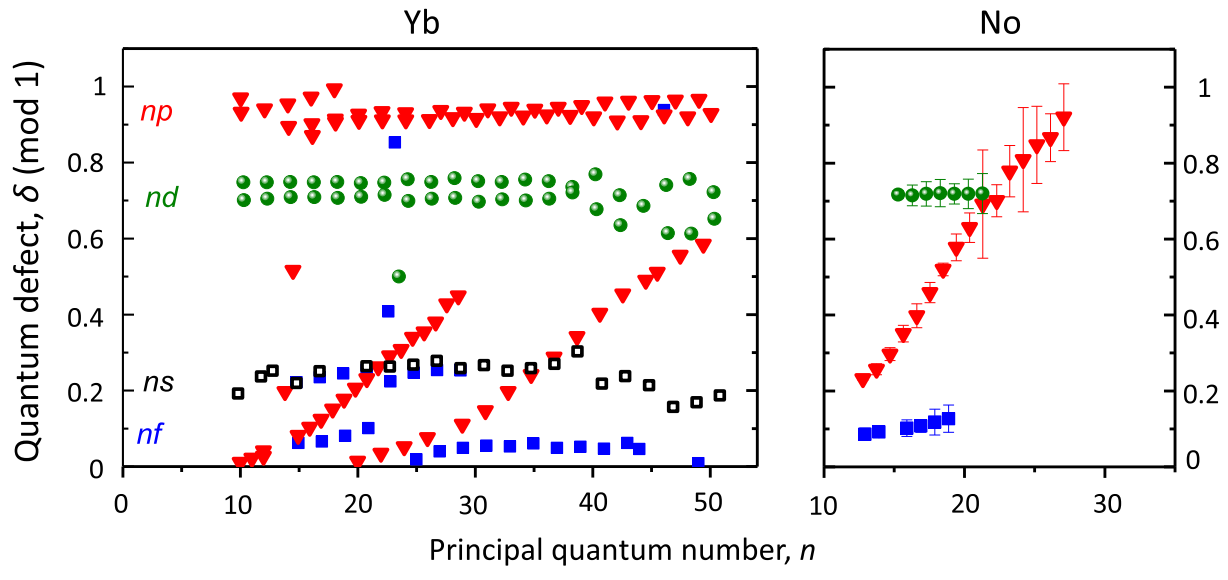

FIG. 3. Quantum defect values for Yb (left panel) and No (right panel). The quantum defects of the three Rydberg series in No and the $n s$ (hollow squares), $n p$ (solid triangles), $n d$ (solid spheres), and $n f$ (solid squares) series in Yb are plotted as a function of the principal quantum number $n$. 
observations with the second, ionizing, laser excitation step delayed with respect to the first revealed that the Rydberg states are excited from two distinct intermediate atomic levels. This is in agreement with the observation of a fast quenching of the ${ }^{1} P_{1}$ state to a longer-lived state due to collisions of the excited atom with the buffer gas [19]. From the convergence of the Rydberg series the energetic position of this long-lived state was derived to be $310_{-8}^{+1} \mathrm{~cm}^{-1}$ below the ${ }^{1} P_{1}$ state. Based on theoretical calculations and the analysis of the quantum defect, a term assignment as ${ }^{3} D_{3}$ was made for this new state, inaccessible by direct laser excitation as the optical transition from the ground state is forbidden.

This work opens the door for forthcoming precision measurements of various atomic and nuclear properties of still heavier elements using laser spectroscopic techniques, for example, the precise determination of the IP for Lr, where the question about the change in the ground-state electronic configuration of Lr, predicted to be [Rn] $5 f^{14} 7 s^{2} 7 p_{1 / 2}$ [34], in contrast to that of its lanthanide homologue lutetium (Lu), [Xe] $4 f^{14} 6 s^{2} 5 d$ can now be addressed. Finally, the ability to optically pump the atoms has the potential to make further transitions accessible, not least for the precise extraction of nuclear properties from isotope shifts and hyperfine structure.

We thank the staff of the GSI ion source and accelerator for preparing the stable ${ }^{48} \mathrm{Ca}$ beam as we are grateful to the staff of the target lab for providing high-quality lead targets. We acknowledge the technical support of J. Maurer, H.-G. Burkhard, D. Racano, L. Braisz, D. Reemts, and I. Kostyuk. This work was supported by the German Federal Ministry of Research under Contracts No. 06MZ169I, No. 06LM236I, FAIR NuSTAR No. 05P09RDFN4, No. 05P12RDFN8, and No. 05P15RDFN1; by the GSI; and by the Helmholtz Association. This project has also received funding from the European Union Horizon 2020 research and innovation programme under Grant Agreement No. 654002 (ENSAR2). D. A. is supported by the European Commission in the framework of CEA-EUROTALENTS (No. PCOFUND GA 2013 600382).

*P.chhetri@gsi.de

†Present address: Max-Planck-Institut für Kernphysik, 69120 Heidelberg, Germany.

[1] L. Öhrström and J. Reedijk, Pure Appl. Chem. 88, 1225 (2016).

[2] P. Schwerdtfeger, L. F. Pašteka, A. Punnett, and P. O. Bowman, Nucl. Phys. A944, 551 (2015).
[3] P. Indelicato, J. P. Santos, S. Boucard, and J.-P. Desclaux, Eur. Phys. J. D 45, 155 (2007).

[4] A. Borschevsky, E. Eliav, M. J. Vilkas, Y. Ishikawa, and U. Kaldor, Phys. Rev. A 75, 042514 (2007).

[5] V. A. Dzuba, M. S. Safronova, and U. I. Safronova, Phys. Rev. A 90, 012504 (2014).

[6] Y. Liu, R. Hutton, and Y. Zou, Phys. Rev. A 76, 062503 (2007).

[7] A. Türler and V. Pershina, Chem. Rev. 113, 1237 (2013).

[8] Y. Nagame, J. V. Kratz, and M. Schädel, Nucl. Phys. A944, 614 (2015).

[9] A. Türler, R. Eichler, and A. Yakushev, Nucl. Phys. A944, 640 (2015).

[10] G. T. Seaborg, Science 104, 379 (1946).

[11] K. Wendt, T. Gottwald, C. Mattolat, and S. Raeder, Hyperfine Interact. 227, 55 (2014).

[12] E. Worden et al., Opt. Soc. Am. B 10, 1998 (1993).

[13] E. Worden and J. Conway, J. Opt. Soc. Am. 69, 733 (1979).

[14] A. Coste, R. Avril, P. Blancard, J. Chatelet, D. Lambert, J. Legre, S. Liberman, and J. Pinard, J. Opt. Soc. Am. 72, 103 (1982).

[15] N. Erdmann et al., J. Alloys Compd. 271-273, 837 (1998).

[16] J. Roßnagel, S. Raeder, A. Hakimi, R. Ferrer, N. Trautmann, and K. Wendt, Phys. Rev. A 85, 012525 (2012).

[17] T. K. Sato et al., Nature (London) 520, 209 (2015).

[18] M. Laatiaoui et al., Nature (London) 538, 495 (2016).

[19] P. Chhetri et al., Eur. Phys. J. D 71, 195 (2017).

[20] S. Hofmann and G. Münzenberg, Rev. Mod. Phys. 72, 733 (2000).

[21] Yu. Ts. Oganessian et al., Phys. Rev. C 64, 054606 (2001).

[22] M. Leino et al., Eur. Phys. J. A 6, 63 (1999).

[23] M. Laatiaoui, H. Backe, M. Block, F.-P. Heßberger, P. Kunz, F. Lautenschläger, W. Lauth, M. Sewtz, and T. Walther, Eur. Phys. J. D 68, 71 (2014).

[24] H. Backe et al., Eur. Phys. J. D 45, 99 (2007).

[25] F. Lautenschläger et al., Nucl. Instrum. Methods, Phys. Res., Sect. B 383, 115 (2016).

[26] M. Laatiaoui, H. Backe, M. Block, P. Chhetri, F. Lautenschläger, W. Lauth, and Th. Walther, Hyperfine Interact. 227, 69 (2014).

[27] V. S. Letokhov, Laser Photoionization Spectroscopy (Academic Press, New York, 1987).

[28] T.F. Gallagher, Rydberg Atoms (Cambridge University Press, Cambridge, England, 1994).

[29] P. Chhetri et al., Acta Phys. Polon. B 49, 599 (2018).

[30] J. Sugar, J. Chem. Phys. 60, 4103 (1974).

[31] P. Camus, A. Débarre, and C. Morillon, J. Phys. B 13, 1073 (1980).

[32] M. Aymar, R. J. Champeau, C. Delsart, and O. Robaux, J. Phys. B 17, 3645 (1984).

[33] U. Fano, C. E. Theodosiou, and J. L. Dehmer, Rev. Mod. Phys. 48, 49 (1976).

[34] A. Borschevsky, E. Eliav, M. J. Vilkas, Y. Ishikawa, and U. Kaldor, Eur. Phys. J. D 45, 115 (2007). 